\title{
THE NATURE OF DOMAIN STABILIZATION IN FERROELECTRIC PEROVSKITES
}

\author{
P. V. LAMBECK and G. H. JONKER \\ Twente University of Technology, P.O.B. 217, 7500 AE Enschede, The Netherlands
}

(Received 23 October 1985; accepted 14 November 1985)

\begin{abstract}
Ferroelectric perovskites, such as $\mathrm{Pb}(\mathrm{Ti}, \mathrm{Zr}) \mathrm{O}_{3}$ and $\mathrm{BaTiO}_{3}$, are subject to ageing effects, caused by a gradual stabilization of the ferroelectric domain structure. We studied the development of this stabilization in $\mathrm{Mn}$-doped $\mathrm{BaTiO}_{3}$ monocrystals having a special kind of domain structure. We concluded from measurements of the growth of the internal bias field $E_{1}$ and the change of the sideways $180^{\circ}$ domain-wall mobility with time, that the fairly strong stabilization in this material originates from volume effects, which implies a gradual reorientation of polar defects with respect to the direction of the spontaneous polarization, both $180^{\circ}$ domain-wall pinning and surface-layer effects being completely absent. A proposal is made about the nature of these defects.
\end{abstract}

Keywords: ferroelectric, barium titanate, ageing, domain-structure stabilization.

\section{INTRODUCTION}

Ageing effects are shown by many materials. They manifest themselves as a spontaneous gradual change of many of their properties with time. In the ageing materials the free enthalpy gradually diminishes caused by various types of processes, for example ion or atom diffusion. The ageing must be regarded as a secondary phenomenon, as it only influences the course of the primary processes which define the macroscopic behaviour of the material. The ageing phenomenon itself has never been applied, but it often determines the applicability of the primary properties.

In this paper we shall concentrate on the origin of the well-known ageing phenomena in ferroelectrics:

(a) a gradual lowering of the dielectric and piezoelectric parameters and of the loss factors $[1,2]$;

(b) a gradual constriction or displacement of the $D-E$ hysteresis loop along the $E$-axis [3-5].

De-ageing or rejuvenation can be brought about by applying a large a.c. voltage to the material (hysteresis-relaxation) [5] or by heating it to above its Curie temperature [6].

Both types of ageing phenomena stem from the same roots, viz. a gradual stabilization of the existing ferroelectric domain structure $[2,5]$. Various stabilization theories have been proposed in the literature [7-11], but nowadays the relative weight (if any) of the corresponding effects is in most materials still unknown. This lack of knowledge applies especially to the ferroelectric perovskites, such as $\mathrm{BaTiO}_{3}$ and $\mathrm{Pb}(\mathrm{Ti}, \mathrm{Zr}) \mathrm{O}_{3}$, which in ceramic form are widely applied in the electronic component industry [12].

This paper reports the first part of a more extensive study of the origin of ageing in these perovskites. In
Section 2 the stabilization models mentioned in the literature are introduced and discussed, resulting in a conception on how to deal with the model study. The preparation of the $\mathrm{Mn}$-doped $\mathrm{BaTiO}_{3}$ crystals is reported in Section 3, together with chemical and physical characteristics of the produced crystals. In Section 4 techniques for conditioning the crystals and measuring their ageing behaviour are described. The experimental results of our study are reported and analysed in Section 5. In Section 6, final conclusions are presented and discussed.

\section{STABILIZATION THEORIES}

The stabilization of the domain structure appears to be strongly dependent on the defect structure. It is well known [13], for example, that, the stabilization is absent or weak in very pure materials and in materials in which positive ions are partly substituted by ions of higher valency. However, substitution by ions of lower valency appears to increase the stabilization enormously. Therefore it is not by accident that all theories have in common that the existing domain structure will be stabilized by changes in the defect distribution. However, they differ greatly in defining the defect driving forces.

\section{(a) The grain-boundary theory $[7,8]$}

In polycrystalline ferroelectrics just after the development of the domain structure, electric fields will originate from the polarization discontinuities at the grain boundaries. The corresponding electric depolarization energy should gradually diminish by diffusion of charged point defects to these boundaries.

\section{(b) Surface-layer model}

We shall take into account another boundary 
model, which we have called the surface-layer model. It is well known that in $\mathrm{BaTiO}_{3}$ crystals, even after etching off a surface-layer of different chemical composition, many properties appear to be thickness dependent $[14,15]$. Callaby [16] explained these effects assuming a "physical" surface-layer about $10 \mu \mathrm{m}$ thick. This layer differs from the bulk of the crystal only by the value of an activation field $\delta$, a parameter defining the field-induced sideways mobility of $180^{\circ}$ domain walls. The origin of this difference is as yet unknown. In the surface-layer model we assume that this $\delta$-value of the surface-layer changes during stabilization.

\section{(c) Domain-wall theory $[5,10]$}

Electric fields of piezoelectric origin or elastic fields arising from the domain walls drive effectively charged or neutral defects to or from the domain walls, so fixing their position.

\section{(d) Volume theory $[4,5,11]$}

Polar lattice defects are reoriented with respect to the direction of the spontaneous polarization. The forces driving the defects may originate from their interaction with either the spontaneous polarization (Lorentz field) or the spontaneous strain. A similar defect repositioning is known to occur in strained $\alpha$-iron [17] and in Co-containing ferrites [18].

All these theories can explain, at least qualitatively, the ageing phenomena [5]. Besides this, experimental evidence exists for all these models. The increase, for example, of the $180^{\circ}$ domain-wall mobility during the field-induced rejuvenation of a $\mathrm{BaTiO}_{3}$ crystal [19] points to a volume model. The etching behaviour of $\mathrm{BaTiO}_{3}$ crystals immediately after the poling process suggests a domain-wall effect [20]. The concentration dependency of the internal field in Al-doped PZT ceramics can be explained by a grain-boundary effect only [5].

In our opinion the following arguments of a quantitative nature will lead to the rejection of the grain-boundary model as being the only source of stabilization:

(a) In this model, in its purest form [7], the internal bias field $E_{i}$, indicating the strength of the domain-structure stabilization, is a real field, finally compensating the depolarization field, and not just a formal one. In fresh material the depolarization field has to be lower than the coercive field $E_{c}$ otherwise the domain structure will be reconstructed. Thus $E_{k}$ cannot exceed $E_{c}$ either. However, for some ceramics $E_{i}$ values obtained are at least twice the $E_{\text {q }}$ value [5].

(b) One can modify the grain-boundary theory by imagining the domain structure being strongly fixed by defects preferentially present in the domain boundaries. Being fixed on the grain boundaries only, the domain structure in the inner of the grain should be fairly free to respond to applied fields [5]. This modification predicts a grain-size dependency of $E$, which, however, has never been reported in the literature.

(c) The rough equality between the hysteresisrelaxation times and the $\rho \varepsilon$-times in Mn-doped PZT ceramics [5] seems to support the grain-boundary model. However, a relation of this nature was not observed in the PZT ceramics produced by Takahashi [8].

We also have a serious objection to the domainwall theory. In a material stabilized by domain-wall pinning an electric field can rearrange the domain structure by nucleation and the subsequent growth of new domains with a favoured $\boldsymbol{P}_{s}$-direction, thus avoiding loosening of the pinned walls, already present. It is known from single crystal studies [21] that the nucleation fields and the fields forcing the walls to move are in the same range.

Volume theory, however, is able to withstand such criticism. Therefore we have formed the opinion that volume effects are most likely to exist, perhaps in some cases accompanied by other effects.

Surveying the literature about ageing we come to the conclusion that the ageing processes are strongly material dependent. Crystal matrix [6], microstructure [5], domain structure [4] and defect structure $[2,5,13]$ all determine the nature and strength of the driving forces and/or the extent to which the system can comply with these forces. This statement contains the key to our model study, which is the examination of the ageing behaviour of systems so arranged that only one or a restricted number of the possible driving forces can be present. For polycrystalline materials this conception is not practicable, but for monocrystals it is. In the latter we can create some special domain structures (see Fig. 1), in this way excluding some sources of ageing.

In a fresh single-domain crystal of high quality, domain walls, mechanical stresses and boundaries with uncompensated polarization charges are miss-

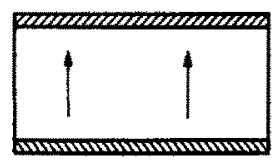

(a)

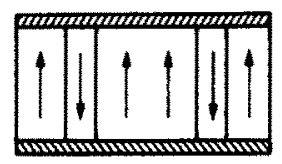

(b)

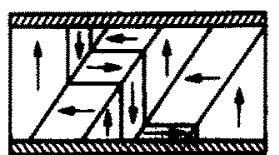

(c)

Fig. 1. Domain structures in a $\mathrm{BaTiO}_{3}$ crystal: a single-domain structure (a), a $c$-domain structure (b) and an $a c$-sandwich domain structure. 
ing. If ageing does exist in such crystals, it could be ascribed to volume or surface-layer effects. By stepwise enlargement of the complexity of the domain structure, other sources can be introduced step by step. In a $c$-domain structure $180^{\circ}$ domain-wall pinning is added, in an ac-sandwich domain structure $90^{\circ}$ wall pinning as well. In crystals with more complicated domain structures stresses will also be present, causing elastic and electric fields.

In our investigations we have used Mn-doped $\mathrm{BaTiO}_{3}$ crystals for the following reasons:

$\mathrm{BaTiO}_{3}$ crystals can be easily produced [22-24]; macroscopic as well as microscopic dielectric behaviour of $\mathrm{BaTiO}_{3}$ crystals is well described in the literature [21];

Mn doping results in a strong stabilization [5, 13]; there is large variability in the defect structure of these crystals [25].

\section{PREPARATION OF SPECIMENS AND SOME OF THEIR CHARACTERISTICS}

In order to obtain $\mathrm{BaTiO}_{3}$ crystals of high quality we used the fux method given by Feltz and Langbein [23]. Because the absence of oxygen is a necessary condition in this technique, all the preliminary handling and the growing process itself have taken place in a nitrogen atmosphere $\left(10 \mathrm{ppm} \mathrm{O}_{2}\right)$. A platinum crucible containing $\mathrm{BaTiO}_{3}(18 \mathrm{~mol} \%), \mathrm{Ba}_{2} \mathrm{TiO}_{4}$ $\left(2 \mathrm{~mol} \%\right.$ ), a small trace of $\mathrm{MnO}$ and $\mathrm{BaCl}_{2}$, the fux $(80 \mathrm{~mol} \%)$ is heated at $1300^{\circ} \mathrm{C}$ for $20 \mathrm{~h}$. The crucible is then slowly $\left(10^{\circ} \mathrm{C} / \mathrm{h}\right)$ cooled down to $1100^{\circ} \mathrm{C}$ at which state the excess liquid is poured off. The crystals thus formed are of the butterfly type just as those produced by the Remeika method [22]. The thickness of the wings (constant in large regions) is in the range of $50-500 \mu \mathrm{m}$, lateral dimensions are in the range of $0.5-1 \mathrm{~cm}$. The absence of a second phase, the monocrystal state and the $\langle 100\rangle$ character of the upper surfaces of the wings were confirmed by means of observations by polarization microscopy.

\section{Chemical composition}

(a) Mn-dope. EMP-analysis revealed an inhomogeneous distribution of the incorporated Mn ions, reflecting the growth behaviour of the crystals [26]. However, after sawing, etching and tempering, crystals showing an almost homogeneous distribution have been obtained. The $\mathrm{Mn}$-concentrations and the $\mathrm{Ba} / \mathrm{Ti}$ ratio as measured by $\mathrm{X}$-ray fluorescence indicate that the $\mathrm{Mn}$ ions are localised on $\mathrm{Ti}$ sites. $\mathrm{Mn}$ concentrations were in the range of $0.1-1.1$ at. $\%$.

The crystals were annealed for several days under well defined conditions (see Table 1), not only to homogenize and relax the stresses induced by sawing, but also to establish defect structures of different types. The sites and valencies of the $\mathrm{Mn}$ ions in the

* To be published elsewhere. three crystal types wcre obtained by EPR measurements.* Some uncertainty remains, because the $\mathrm{Mn}^{3+}$ ion does not manifest itself in the spectra. We have also calculated the percentage of $\mathrm{Mn}$ ions present in the $2^{+}, 3^{+}$and $4^{+}$states from the defect-equilibrium data given by Hagemann [25]. They agree roughly with our experimental data (see Table 2).

(b) Impurities. Impurities in our crystals originate from the starting chemicals and the materials employed in the growing process. The presence of $\mathbf{S r}^{2+}$ and $\mathrm{Ca}^{2+}$ ions $(<0.1 \%)$ is not alarming, because these lons, incorporated on $\mathrm{Ba}^{2+}$ sites are not significant for the ageing. $\mathrm{Fe}$ and $\mathrm{Cl}$ concentrations appeared to be under the limits of detection (X-ray fuorescence) being 0.01 and 0.1 at. $\%$, respectively. Neutron activation analysis showed a Pt concentration of about 0.001 at. $\%$.

(c) Physical characteristics. Non-stabilized crystals provided with liquid electrodes show an almost rectangular hysteresis loop, the spontaneous polarization $P_{s}$ being $26 \pm 2 \mu \mathrm{C} / \mathrm{cm}^{2}$; the resistivities of all types of crystals were larger than $10^{14} \Omega \mathrm{m}$.

The linear decrease of the Curie temperature $T_{\text {, }}$ (determined by microscopial observation) with increasing dope concentration (see Fig. 2) indicates the homogeneity of the $\mathrm{Mn}$ incorporation. The high $T_{\text {c }}$ value of the undoped crystals $\left(128^{\circ} \mathrm{C}\right)$ illustrates their good quality.

\section{EXPERIMENTAL}

\section{(a) Measuring methods}

Mainly traditional measuring methods were employed. The internal bias field $E_{n}$, indicating the strength of the stabilization, was obtained from $\mathrm{d} P / \mathrm{d} t-E$ hysteresis loops [5] (see Fig. 3), registered by means of a Sawyer-Tower circuit [27]. Fieldinduced movements of $180^{\circ}$ domain walls were observed by means of a polarization microscope [28], their velocities being obtained by film camera registrations.

\section{(b) Required domain structures}

In order to produce non-stabilized single- or $c$-domain structures we proceeded in the following way:

After d.c. poling in water the crystals were partly electroded by applying droplets of a saturated solution of $\mathrm{LiCl}$ in water to their upper and lower faces. The use of such liquid electrodes enables mechanical stresses in the crystal, originating from clamping by

Table 1. The $\mathrm{Ba} / \mathrm{T}$ i ratio and the $\mathrm{Mn}$ concentration $c_{\mathrm{Mn}}$ in some Mn-doped $\mathrm{BaTiO}_{3}$ crystals

\begin{tabular}{|c|c|c|c|}
\hline & $\mathrm{Ba} / \mathrm{Ti}[\mathrm{atz} / \mathrm{atg}]$ & $c_{\mathrm{Mn}}$ & {$[\operatorname{atc}]$} \\
\hline crystal 1 & $1.007 \pm 0.003$ & 0.5 & \pm 0.1 \\
\hline crystal 2 & $1.010 \pm 0.002$ & 1,1 & \pm 0.1 \\
\hline
\end{tabular}


Table 2. Annealing conditions, Mn valency and compensation mechanism of the three types of Mn-doped $\mathrm{BaTiO}_{3}$ crystals

\begin{tabular}{|c|c|c|c|c|c|c|c|c|c|}
\hline temp. & \multicolumn{2}{|c|}{ gas atmosphere } & \multicolumn{3}{|c|}{ experimental* } & \multicolumn{3}{|c|}{ calculated ** } & Compensation mechanism \\
\hline$[* \mathrm{c}]$ & type & $\mathrm{po}_{2}[\mathrm{~atm}]$ & $\mathrm{Mn}^{4+}$ & $\mathrm{Mn}^{3+}$ & $\mathrm{Mn}^{2+}$ & $\mathrm{Mn}^{4+}$ & $\mathrm{Mn}^{3+}$ & $\mathrm{Mn}^{2+}$ & \\
\hline 700 & $3 \times x$ & 0.2 & + & 2 & - & 948 & 68 & - & {$\left[\mathrm{Mn}^{3+}\right]_{\mathrm{T}_{2}} 4+=2\left[\mathrm{v}_{\mathrm{O}}^{2+}\right]$} \\
\hline 850 & $\mathrm{~s}_{2}$ & $10^{-5}$ & - & + & - & 408 & 608 & - & {$\left[\mathrm{Mn}^{3+}\right]_{\mathrm{T} 2}{ }^{4+}=2\left[\mathrm{vg}^{2+}\right]^{2+}$} \\
\hline 850 & $\mathrm{~N}_{2} / \mathrm{H}_{2}$ & $10^{-22}$ & - & 2 & + & - & - & 1008 & {$\left[\mathrm{Mn}^{2+}\right]_{\mathrm{T}_{2}}{ }^{4+}=\left[\mathrm{v}_{\mathrm{O}}^{2+}\right]$} \\
\hline
\end{tabular}

* Deduced from EPR measurements; +: present, -: absent, ?: presence uncertain.

** Calculated from the defect-equilibria data given by Hagemann [25].

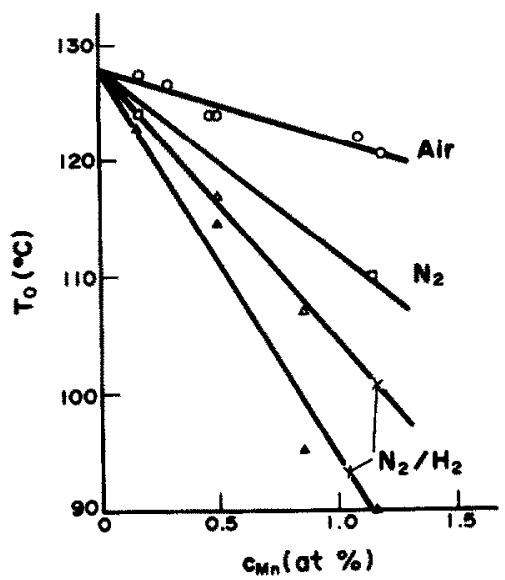

Fig. 2. The paraelectric-ferroelectric $(p \rightarrow f)$ phasetransition temperature $T_{0}$, as a function of the Mn concentration $c_{M n}$ in crystals annealed in air $(O)$, in nitrogen ( $\square$ ) and in a nitrogen/hydrogen mixture $(\Delta: p \rightarrow f ; \Delta: f \rightarrow p)$. the electrodes, and fatigue effects [21] to be avoided. These partly electroded crystals were installed in a Sawyer-Tower circuit in order to observe the hysteresis loop. By means of hysteresis-relaxation the $D-E$ loop finally attains a symmetrical shape, indicating the complete destabilization of the electroded volume. At a moment that $P=P_{s}$ the applied voltage is switched off, $P_{s}$ being parallel to the $P_{s}$-direction created by the previous water poling. Hence the destabilized volume consists of one domain, $180^{\circ}$ domain walls being absent at the boundaries. On the other hand, by switching off at $P=0$, a destabilized $c$-domain structure is obtained. The correctness of the procedure is proved by microscopic observation of the static and dynamic properties of the domain structure.

An a.c. domain structure can be obtained by slowly cooling down the crystal from the paraelectric to the ferroelectric phase, maintaining a small onedimensional temperature gradient [29]. Rapid cool-
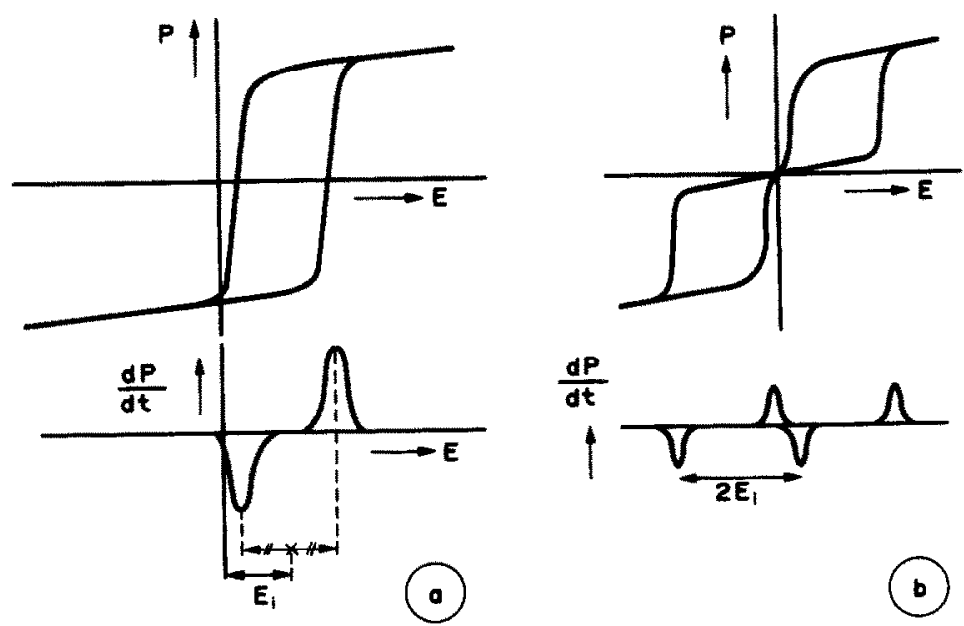

Fig. 3. Hysteresis loops of a crystal aged in a single-domain state (a) or a $c$-domain state (b). 


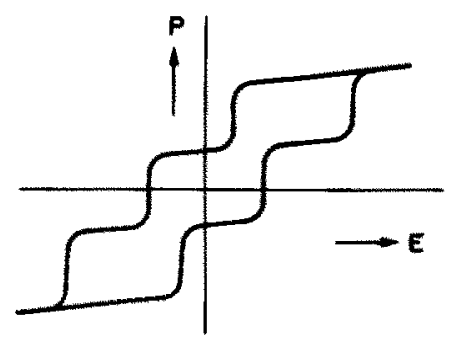

Fig. 4. $P-E$ triple hysteresis loop.

ing results in a more complicated domain structure. Because stabilization starts as soon as the ferroelectric phase is reached, the last two methods do not produce crystals destabilized at chosen temperatures.

\section{RESULTS AND ANALYSIS}

For experimental reasons we have restricted our work to single- and $c$-domain structure crystals.

(1) Just as in poled polycrystalline materials [5] in the single-domain crystals a gradual displacement of the $D-E$ hysteresis loop along the $E$-axis could be observed during the ageing. Analysis of the ageing processes shows that the internal bias field $E_{3}$ appears to develop like the sum of a small number of relaxation processes:

$$
E_{\mathrm{t}}(t)=\sum_{\gamma} E_{i 0_{y},}\left(1-\mathrm{e}^{-4 / \tau_{\gamma}}\right)
$$

The values of the various parameters appear to be strongly dependent on the Mn concentration, the tempering conditions and the temperature during ageing. Some data are given in Table 3 .* $^{*}$

At a first glance it would seem correct to immediately conclude that this ageing originates from volume or surface-layer effects. However, a complication did arise. Observations by polarization microscopy revealed that in our single-domain crystals the polarization reversal always started from the same points, which were just those points where the domains with polarization antiparallel to the applied field were contracted during the preceding poling process. This suggests that non-reversed domains of submicroscopic size might be left over after the poling process. Therefore a pinning of the remaining $180^{\circ}$ domain walls as the origin of the observed stabilization cannot be excluded.

To solve this ambiguity we performed the following experiments:

At a given time, $t=0$, a square wave a.c. voltage was applied to a completely destabilized $c$-domain crystal. The wave period $T$ was chosen to be small as compared with the relaxation times of the stabilization processes. The amplitude was so chosen

\footnotetext{
* Full details will be published elsewhere.
}

that it would only permit a partial polarization reversal. Microscopic observations established that the reversal was only brought about by sideways movement of the existing domain walls. Assuming stabilization by volume or surface-layer effects, the electroded part of the crystal can now be divided into three regions:

regions stabilizing in the $+P_{s}$ or $-P_{s}$ state;

regions where all stabilization is impeded by the continuous polarization reversals. For a field-induced polarization reversal of the stabilized regions, nonpinned $180^{\circ}$ domain walls are available.

At a given time, $t_{1}$, we replace the square voltage by a triangular a.c. voltage large enough to show the full hysteresis loop, or by a d.c. voltage resulting in a slow penetration of the domain walls into the stabilized regions. In the former case, a triple loop was observed (see Fig. 4), showing an $E_{i}\left(t_{1}\right)$ value equal to that of the crystal stabilizing in a singledomain state. In the latter case the domain walls appeared to move with a position-independent velocity, this velocity being dependent on the stabilization time $t_{1}$ and, as expected, on the strength of the applied field (see Fig. 5a). These observations unambiguously lead to the conclusion that the observed stabilization is caused by volume effects or surfacelayer effects.

(2) In order to discriminate between volume and surface-layer effects we proceeded in two different ways:

(a) Both effects can be easily incorporated into the microscopic model for field-induced sideways $180^{\circ}$ domain-wall movement given by Miller and Weinreich [30] (see Appendix). In the case of volume effects the well-known relation for the wall velocity $v,[21]$

$$
v=v_{\infty} \mathrm{e}^{-\delta / E_{a}}
$$

has to be transformed into

$$
v=v_{\infty} \mathrm{e}^{-\delta\left[\left[E_{d}-E_{l}(t)\right]\right.},
$$

and in the case of a surface-layer effect into

$$
v=v_{\infty} \mathrm{e}^{-\left[\alpha(n) \varepsilon_{a}\right\rfloor}
$$

where $E_{\alpha}$ is the applied field. The parameter $\delta$ is usually called the activation field.

The surface-layer model predicts a linear in $v-\left(1 / E_{a}\right)$ relation (with $t_{1}$ as a parameter). This linearity is affirmed by the experimental data (see Fig. 6). However, contrary to this relation it is not the $\delta$-parameter that appears to be time-dependent but the $v_{\infty}$-parameter (see Table 4). Because of this inconsistency we reject the proposed surface-layer model. In the evaluation of the correctness of eqn (2) i.e. the relation based on the volume theory, advantage can be taken of one of the experimental data 


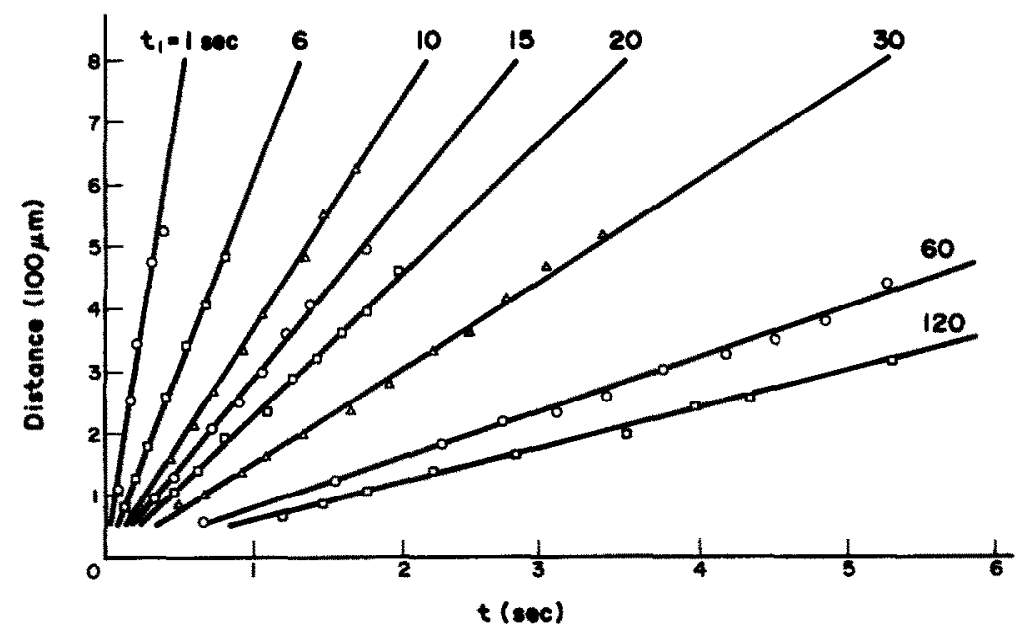

Fig. 5(a). The sideways $180^{\circ}$ domain-wall movement, given as the distance covered vs time, in a crystal annealed in air, containing 0.3 at. $\% \mathrm{Mn}$ after various stabilization times $t_{1}$. Applied field $E_{a}=193.3 \mathrm{~V} / \mathrm{cm}$; temperature $25^{\circ} \mathrm{C}$.

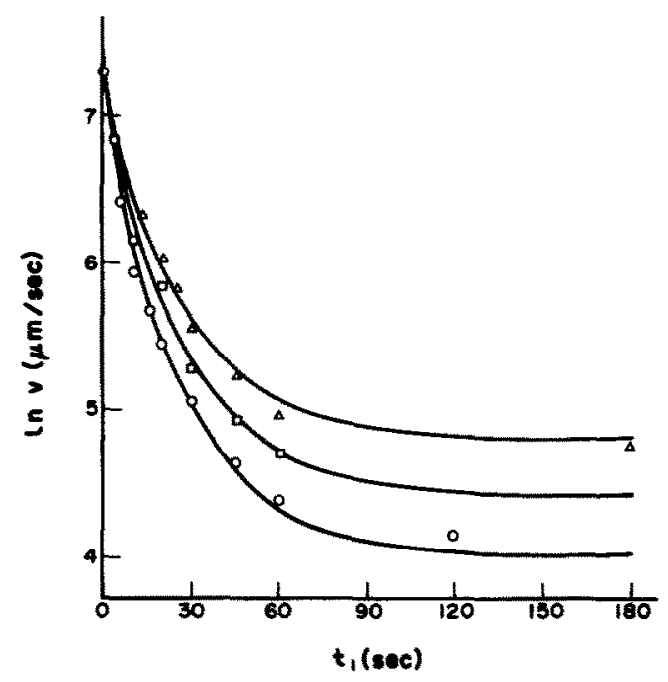

Fig. $S(b)$. The sideways $180^{\circ}$ domain-wall velocity $v$ as a function of the stabilization time $t_{1} .0, \square, \Delta$ : experimental points with $E_{a}=193.3 \mathrm{~V} / \mathrm{cm}, 200.0 \mathrm{~V} / \mathrm{cm}$ and $206.7 \mathrm{~V} / \mathrm{cm}$, respectively. Drawn lines represent calculated values (see text) presented in Table 3 , viz. that in crystals annealed in air $E_{i}$ is built up as the sum of two relaxation processes:

$$
E_{N}(t)=E_{i 0.1}\left(1-\mathrm{e}^{-t / t_{1}}\right)+E_{i 0.2}\left(1-\mathrm{e}^{-t / \tau_{2}}\right) .
$$

By means of a parameter-evaluation method we have determined the parameter set that results in the most linear

$$
\begin{array}{ll}
\ln v-1 /\left[E_{a}-E_{i}(t)\right] \text { plot (see Fig. 7) } \\
\tau_{1}=4 \pm 1 \mathrm{~s} & E_{i 0,1}=33 \pm 3 \mathrm{~V} / \mathrm{cm} \\
\tau_{2}=25 \pm 1 \mathrm{~s} & E_{00,2}=65 \pm 2 \mathrm{~V} / \mathrm{cm} .
\end{array}
$$

The $v\left(E_{a}, t_{1}\right)$ curves, calculated by substituting these parameter values into eqn (2) are presented in Fig. 5(b). They fit the experimental points fairly well.

The correctness of this latter analysis is strongly supported by the data deduced from studies of the

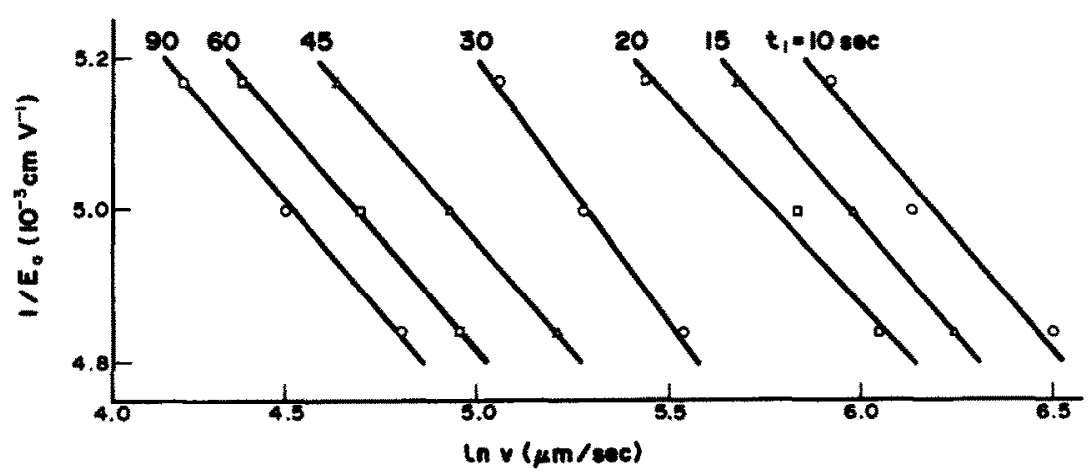

Fig. 6. The sideways $180^{\circ}$ domain-wall movement. $1 / E_{a}-\ln v$ plots at various stabilization times $t_{1}$. 
Table 3. Some ageing parameters of $\mathrm{BaTi}_{099} \mathrm{Mn}_{001} \mathrm{O}_{3}$ crystals aged at $45^{\circ} \mathrm{C}$

\begin{tabular}{|c|c|c|c|c|}
\hline \multicolumn{3}{|c|}{ arnesing conditions } & \multirow{2}{*}{$\sum_{i 0, \gamma}$} & \multirow{2}{*}{$\begin{array}{l}\text { processes } \\
\text { completed } \\
\text { after }\end{array}$} \\
\hline temperature & \multicolumn{2}{|c|}{ gas atmonphere } & & \\
\hline$\left[{ }^{\circ} \mathrm{C}\right]$ & type & $\mathrm{pO}_{2}$ [atm] & {$[\mathrm{kv} / \mathrm{cm}]$} & \\
\hline 700 & $a 1 x$ & 0.2 & 0.35 & $20 \mathrm{~m} 2 \mathrm{n}$. \\
\hline 850 & $\mathrm{~N}_{2}$ & $10^{-5}$ & 7.2 & 1 week \\
\hline 850 & $\mathrm{~N}_{2} / \mathrm{H}_{2}$ & $10^{-22}$ & 5.5 & 1 day \\
\hline
\end{tabular}

Table 4. $v_{\infty}$ and $\delta$ values at various stabilization times $t_{i}$, as calculated from eqn (3)

\begin{tabular}{|l|c|c|}
\hline $\begin{array}{l}t_{1} \\
{[\mathrm{sec}]}\end{array}$ & $\begin{array}{c}\delta_{\text {off }} \\
{[\mathrm{KV} / \mathrm{cm}]}\end{array}$ & $\begin{array}{l}\text { ln } \mathrm{v}_{\infty} \\
{[\mathrm{m} / \mathrm{sec}]}\end{array}$ \\
\hline 10 & 1.72 & 14.8 \\
15 & 1.70 & 14.5 \\
20 & 1.82 & 14.9 \\
30 & 1.43 & 12.4 \\
45 & 1.73 & 13.6 \\
60 & 1.76 & 13.2 \\
90 & 1.70 & 13.6 \\
\hline
\end{tabular}

hysteresis-relaxation process in the same crystal.* These also reveal two processes, a slower one showing $E_{i 0}=69 \pm 3 \mathrm{~V} / \mathrm{cm}$ and $\tau=30 \pm 3 \mathrm{~s}$ and a faster one showing $E_{10}=35 \pm 5 \mathrm{~V} / \mathrm{cm}$. So we conclude that there is only a volume effect and we reject the surface-layer theory.

(b) A completely stabilized Mn-doped singledomain crystal of the nitrogen-tempered type was etched to half its thickness, the etching time being

* To be published elsewhere. much shorter than the relaxation times of the stabilization processes. Thus if any surface layer effect might exist, this layer and the corresponding stabilization must also be removed by etching. However, the hysteresis loops before and after etching reveal almost equal $E_{i}$ values. This experiment also points out the non-existence of a surface-layer effect in these crystals.

(3) The $E_{i}$ development in a crystal appears to show the same time-dependency either in singledomain or in $c$-domain states. This indicates the absence of $180^{\circ}$ domain-wall pinning effects.

\section{DISCUSSION}

From the above data we have concluded that the fairly strong domain structure stabilization in $\mathrm{Mn}$ doped single-domain and $c$-domain $\mathrm{BaTiO}_{3}$ crystals is due to volume effects, both $180^{\circ}$ domain-wall pinning and surface-layer effects being completely absent.

The relative strength of the stabilization of crystals of different defect structures agrees very well with the empirical statement that only ions having a valency lower than that of the ions they substitute will be effective for the stabilization. In the crystals tempered at $\mathrm{pO}_{2}=10^{-5} \mathrm{~atm}$ or $10^{-22} \mathrm{~atm}$ all $\mathrm{Mn}$ ions substituting the $\mathrm{Ti}^{4+}$ ions have in fact a lower valency. In the crystals tempered in air, however, the majority of the $\mathrm{Mn}$ ions are tetravalent and thus without significance for the stabilization. Hence the empirical statement predicts a weaker stabilization in the latter crystals as is observed experimentally. Usually the Mn-doped PZT materials are produced under conditions resulting in a mixture of $\mathrm{Mn}^{3+}$ and $\mathrm{Mn}^{4+}$ ions. We expect that tempering at lower oxygen pressures will result in an increase of the stabilization in these materials too.

We expect the volume effects to be due to a reorientation of $\mathrm{Mn}^{2+}-\mathrm{V}_{0}^{2+}$ and $\mathrm{Mn}^{3+}-\mathrm{V}_{0}^{2+}$ associates

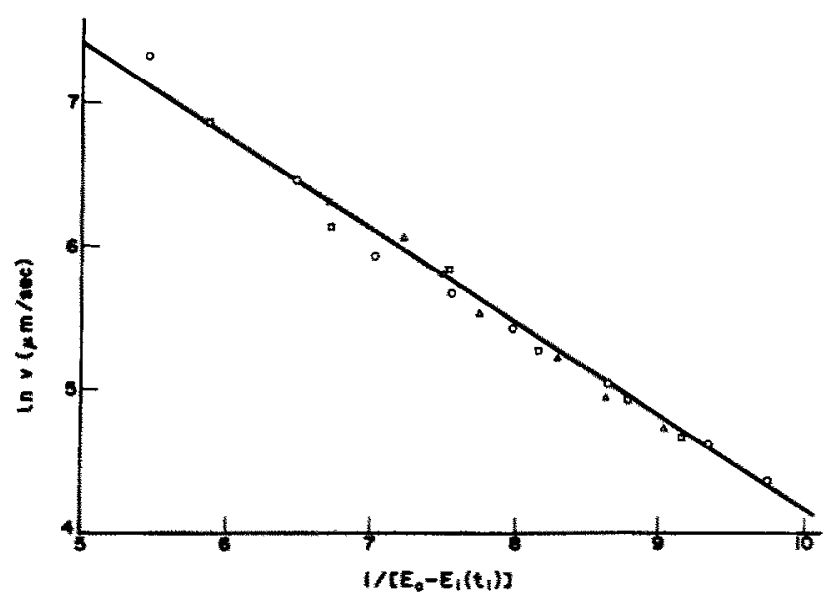

Fig. 7. The sideways $180^{\circ}$ domain-wall movement. Drawn line represents the $\ln v\left(E_{t,}, t_{1}\right)-1 /\left[E_{u}-E_{i}\left(t_{1}\right)\right]$ relation as calculated by substituting eqn (5) into eqn (4). $0, \square, \triangle$ : experimental $\ln v\left(E_{a}, t_{1}\right)$ values for $E_{a}=193.3 \mathrm{~V} / \mathrm{cm}, 200.0 \mathrm{~V} / \mathrm{cm}$ and $206.7 \mathrm{~V} / \mathrm{cm}$, respectively. 
in respect of the existing direction of the spontaneous polarization. This expectation is based on the following arguments:

(a) The compensation mechanisms indicate that the $\mathrm{V}_{0}^{2+}$ concentration is in the same range as the $\mathrm{Mn}^{3+}$ or $\mathrm{Mn}^{2+}$ concentrations. Favoured by the Coulomb interaction, the effectively negatively charged $\left[\mathrm{Mn}^{2+}\right]_{\mathrm{T}^{4+}}$ or $\left[\mathrm{Mn}^{3+}\right]_{\mathrm{T}_{1}^{4+}}$ point defects will associate partly with the effectively positively charged point defect $\mathrm{V}_{0}^{2+}$. Associates of a similar type have been proved to be present in $\mathrm{Fe}^{3+}$-doped $\mathrm{SrTiO}_{3}$ [31].

(b) These defect associates, showing a dipole character, can be oriented in three different ways, the dipole being parallel, antiparallel or perpendicular to the spontaneous polarization. These different orientations will show different energies, probably due to the interaction of the defect dipole and the spontaneous polarization of the crystal matrix.

(c) The defect dipole can be easily reoriented by a jump of an oxygen ion next to the $\mathrm{Mn}$ ion into the oxygen vacancy.

(d) We rule out bare oxygen vacancies as stabilization-active defects: if they were the stabilizers, the crystals containing $\mathrm{Mn}^{2+}$ and $\mathrm{Mn}^{3+}$ might show the same time dependency of $E_{i}$ during ageing, but this is contradicted by the experiments.

We are of the opinion that defect dipoles of this type will play an important role in the photorefractive effects as well.

\section{REFERENCES}

1. See, for example, Martin H. J., Die Ferroelektrika. Akad. Verlag, Leipzig (1964).

2. Hagemann H. J., J. Phys. C, Solid State Phys. 11, 3333 (1978)

3. Kambe K. J., J. Phys. Soc. Jpn 8, 15 (1953)

4. Unruh H. G., Z. Angew. Phys. 16, 315 (1963).

5. Carl K. and Härdtl K. H., Ferroelectrics 17, 47 (1978).

6. McQuarrie M. C. and Buessem W. R., Am. Ceram. Soc. Bull. 34, 402 (1955).

7. Okasaki K. and Sakata K., Electrotechn. J. Jap. 7, 13 (1962).

8. Takahashi M., Jap. J. Appl. Phys. 9, 1236 (1970)

9. Thomann H., Ferroelectrics 12, 141 (1972),

10. Miserova A., Sov. Phys. Sol. State 2, 1160 (1960).

11. Jonker G. H., J. Am. Ceram. Soc. 55, 57 (1972).

12. Härdtl K. H., Ferroelectrics 12, 9 (1976)

13. See, for example, Jaffe B., Cook W. R. and Jaffe H., Piezaelectric Ceramics. Academic Press, New York (1971)

14. Merz W. J., J. Appl. Phys. 27, 938 (1956).

15. Miller R. C. and Savage A., J. Appl. Phys. 32, 714 (1961).

16. Callaby D. R., J. Appl. Phys. 36, 2751 (1965)

17. See, for example, Fast J. D., Entropy. Centens Uitg. Hilversum, Netherlands (1948).

18. de Lau J. G. M., Ph.D. thesis, TH Eindhoven, Netherlands (1975).

19. Miller R. C. and Savage A., J. Appl. Phys. 31, 668 (1960).

20. Stadler H. L., J. Appl. Phys. 34, 570 (1963)

21. See, for example, Fatuzzo E. and Merz W. J., Ferroelectricity, North-Holland, Amsterdam (1967).

22. Remeika J. P., J. Am. Ceram. Soc. 76, 940 (1954).
23. Feltz A. and Langbein H., Krist. Techn. 6, 359 (1971)

24. Belruss V., Kalnays J. and Linz A., Mater. Res. Bull. 6, 899 (1971).

25. Hagemann H. J., Ph.D. thesis, TH Aachen (1980).

26. Perry F. W., Hutchins G. A. and Cross L. E., Mater Res. Bull. 2, 409 (1967)

27. Sawyer C. B. and Tower C. H., Phys. Rev. 35, 269 (1930).

28. Miller R. C. and Savage A., Phys. Rev. Lett. 2, 294 (1959).

29. Burfoot J. C. and Parker T. J., Br. J. Appl. Phys. 17, 207 (1966).

30. Miller R. C. and Weinreich G., Phys. Rev. 117, 1460 (1960).

31. Kirckpatrick E. S., Müller K. A. and Rubins R. S., Phys. Rev. 135, 341 (1964)

32. See, for example, Brice J. C., The Growth of Crystals from Liquids, p. 103, North-Holland, Amsterdam (1973).

\section{APPENDIX}

Incorporation of volume and surface-layer effects in the Miller-Weinreich model

According to the theory of Miller and Weinreich [30] the velocity of the field-induced sideways movement of $180^{\circ}$ domain walls is completely governed by nucleation, i.e. nucleation of small domains with $P_{s}$ parallel to the field, next to a perfectly smooth domain wall. Hence it is an analogy of the crystal growth on perfect sharp singular interfaces [32]. Just as in all nucleation phenomena the nucleation probability will be proportional to $\exp (-\Delta F / k T)$, in which $\Delta F$ is the increase of free enthalpy connected with the building up of the critical nucleus. In this case the wall velocity can be expressed as

$$
v=v_{x} \exp [-\Delta F /(k T)]
$$

In a non-stabilized crystal, $\Delta F$ consists of three terms. The first reflects the interaction between the applied field $E_{a}$ and the spontaneous polarization $P_{5}$, the interaction energy being $-2 E_{a} P_{s} V$, where $V$ is the volume of the critical nucleus. The second term shows the increase in domain-wal energy caused by the enlargement of the total wall surface. The third term refiects the depolarization energy. We emphasize that only the first term is a function of $E_{\alpha}$. From the complete expression for $\Delta F$ Miller and Weinreich finally deduced the relation

$$
v=v_{x} \exp \left(-\delta / E_{q}\right)
$$

$\delta$ being a complicated function of a number of material constants and the temperature.

In our opinion, the best way to introduce the volume effect stabilization in this scheme is by adding a stabilization term $2 E,(t) P_{s} V$, so that the $\Delta F$ value of a stabilized erystal can be obtained from the original one by simply replacing $E_{u}$ by $\left[E_{a}-E_{i}(t)\right]$. This leads to

$$
v=v_{x} \exp \left\{-\delta /\left[E_{a}-E_{\iota}(t)\right]\right\}
$$

As to the surface-layer model we start from the $v$ relation obtained by Callaby [16]:

$$
v=v_{x} \exp \left[-\frac{\delta_{B}+\frac{2 l}{d}\left(\delta_{L}-\delta_{B}\right)}{E_{a}}\right]
$$


where $l$ and $d$ are the thickness of the bilk and the surface layer, respectively and $\delta_{l}$ and $\delta_{d}$ their activation fields. Because surface layer and bulk only differ in the activation field, a stabilization will most likely manifest itself as a change in this parameter. Thus relation (A.4) will transform into

$v=v_{x} \exp \left[-\delta(t) / E_{a}\right]$

(A.5) 